Linköping Studies in Science and Technology.

Thesis No. 1639

\title{
Goal Function Approach to Growth and Remodeling of Arteries
}

\section{Ganarupan Satha}

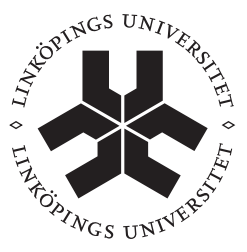

\section{Linköping University INSTITUTE OF TECHNOLOGY}

Department of Management and Engineering, Division of Mechanics Linköping University, SE-581 83, Linköping, Sweden 
Printed by:

LiU-Tryck, Linköping, Sweden, 2014

ISBN 978-91-7519-433-2

ISSN 0280-7971

Distributed by:

Linköping University

Department of Management and Engineering

SE-581 83, Linköping, Sweden

\section{(C) 2014 Ganarupan Satha}

This document was prepared with $\mathrm{LT}_{\mathrm{E}} \mathrm{X}$, February 3, 2014

No part of this publication may be reproduced, stored in a retrieval system, or be transmitted, in any form or by any means, electronic, mechanical, photocopying, recording, or otherwise, without prior permission of the author. 


\section{Preface}

This licentiate thesis was carried out at the Division of Mechanics, Department of Management and Engineering, Linköping University and the project was funded by the Swedish Research Council. First of all I would like express my deepest appreciation for my main supervisor, Professor Anders Klarbring, for giving me the opportunity to study this very interesting research topic. I greatly admire your experience and deep knowledge of your research field. Your help and guidance has been very useful in my studies. I am also deeply indebted to my co-supervisor Associate Professor Stefan B. Lindström. Your comments, suggestions, guidance and contributions have been very valuable throughout the course of my study. I have learned a lot from you and I am inspired by your teaching, your way of explaining things and your way of thinking. It has been very fun to work and write scientific research articles with the both of you. I would also like to thank cosupervisor Associate Professor Jonas Stålhand for useful comments on my work. I am also thankful for the company and support of the staff at the Division of Mechanics, who have made life at work fun and pleasant.

I am also very grateful to my family, without whose moral support and warm encouragement I could not have gone to work with confidence every day. Even if you live far away from me, you are always present in my heart. Many thanks also go to Anders Wirén, Jonas Nilsson and Akiltharushan Umaganthan, as well as numerous other friends, for making life on this earth brighter and more stable.

Ganarupan Satha

Linköping, January 2014 



\section{Abstract}

In this thesis we develop a new goal function approach to investigate stability of the growth processes in blood vessels and cost-optimal composition and geometry of these vessels. In the vascular system of a healthy individual, the living composition of the arterial wall must regenerate and remodel continuously during the entire lifetime to maintain itself. In some cases the system destabilizes due to disease, injury or other complex processes. To understand how and when this happens, several mathematical models have been developed. These models have included an evolution equation for mass fractions of the vessel wall, describing how the vessel develops from an actual state to a target state. These works are based on constrained mixture theory (CMT), which takes care of production and removal of arterial constituents. The cost-optimal design of blood vessels has been studied previously by Murray.

The aim of this thesis is to contribute to stability analyses of the growth process by formulating a new goal function approach, making it possible to examine under which conditions instability arises. We also aim to analyze changes in the optimum material composition and geometry of the vessel wall, using a more realistic, nonlinear material model.

The blood vessel is modeled as a thin-walled tube and the constituents that form the vessel wall are assumed to deform together (CMT). The growth dynamics of the composite material of the vessel wall is described by an evolution equation, where the effective area of each constituent changes in the direction of steepest descent of a goal function. This goal function is formulated in such way that the constituents grow toward a target potential energy and a target composition. The response of the evolution equation is simulated for several different material models. These simulations suggest that elastin-deficient vessels are more prone to growth instability, but that increased vessel stiffness gives a more stable growth process. Another important finding is that an increased rate of degradation of materials impairs growth stability.

By extending Murray's law to include effects of nonlinear mechanics of the artery wall and a growth and remodeling mechanism based on CMT, and at the same time having the system satisfy an equilibrium equation, we study cost-optimal compositions and geometries of the vessel wall. This gives new insight into the wall's architecture under optimal conditions. 



\section{List of Papers}

This thesis is based on these two appended papers:

I. A goal function approach to remodeling of arteries uncovers mechanisms for growth instability

II. Extension of Murray's law including nonlinear mechanics of a composite artery wall

The papers have been reformatted to fit the layout of this thesis. 



\section{Contents}

$\begin{array}{ll}\text { Preface } & \text { iii }\end{array}$

Abstract $\quad$ v

List of Papers vii

$\begin{array}{ll}\text { Contents } & \text { ix }\end{array}$

1 Background 1

1.1 Vascular system . . . . . . . . . . . . . . . . . . . . . . . . . . 1

1.2 Structure of the arterial wall . . . . . . . . . . . . . . . . . . 1

1.3 Growth and remodeling in blood vessels . . . . . . . . . . . . 3

2 Motivation 5

3 Methods $\quad 7$

3.1 Growth and remodeling control system . . . . . . . . . . . . . 7

3.2 Thin-walled tube . . . . . . . . . . . . . . . . . . 7

3.3 Constrained mixture theory . . . . . . . . . . . . . . . . . . . 8

3.4 Local remodeling of arteries . . . . . . . . . . . . . . . . . . . . . . . . . . 9

3.5 Target geometry and composition . . . . . . . . . . . . . . . 10

$\begin{array}{llr}4 & \text { Results and discussion } & 11\end{array}$

5 Conclusions $\quad 15$

Bibliography 19

Paper I: A goal function approach to remodeling of arteries uncovers mechanisms for growth instability . . . . . . . . . . . . . . 23

Paper II: Extension of Murray's law including nonlinear mechanics of a composite artery wall . . . . . . . . . . . . . . . . 41 



\section{Background}

\subsection{Vascular system}

The primary role of the vascular system of vertebrate animals is to transport blood through the body. The blood transports nutrition, oxygen and hormones to organs and cells, and carbon dioxide and other waste products away from the cells, in order to maintain a steady state [1].

The vascular system consists of a complex network of arteries, arterioles, capillaries, veins and venules. The main purpose of the arteries and arterioles (small arteries) is to carry oxygenated blood away from the heart to the body [1]. The main purpose of the veins and venules (small veins) is to carry blood back to the heart, for further transportation to the lungs where it is re-oxygenated. High pressure blood is carried by arteries and low pressure blood is carried by veins. Normally, the wall of arteries is thicker than that of veins [2]. At the junction between arteries and veins are the capillaries, the place where the blood delivers its oxygen and nutrients to the surrounding tissue, and takes up carbon dioxide and waste products from the tissue.

\subsection{Structure of the arterial wall}

Arteries are subdivided into two types: elastic and muscular. Elastic arteries, for instance the aorta, have a larger diameter and are located closer to the heart. Muscular arteries, for instance the femoral, celiac and cerebral arteries, are located at the periphery [2].

The wall of arteries consists of three layers: intima, media and adventitia (see Fig. 1). Building blocks that make up the vascular wall are endothelial cells, elastin, collagen and vascular smooth muscle cells [1].

The intima is the inner layer of the arterial wall. It is composed of a single layer of endothelial cells, basal lamina and a mixed material subendothelial layer [2]. The endothelium is the thin layer of cells forming an interface between circulating blood in the lumen and the rest of the vessel wall. The transition between intima and media is called the internal elastic lamina [2]. The media is the middle layer 


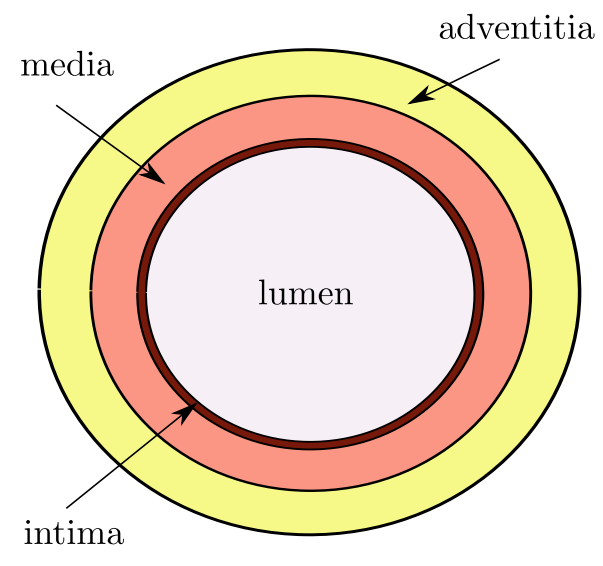

Figure 1: The three layers of the arterial wall: intima (inner layer), media (middle layer) and adventitia (outer layer).

of the walls of arteries. It is composed of vascular smooth muscle, collagen and elastin, which together are oriented as a fibrous helix around the vessel $[2,3]$. The elastin content is higher closer to the heart [2]. The adventitia is the outer layer of arteries and it is composed of connective tissues, including collagen, elastin and fibroblasts $[2,3]$. The connective tissues are composed of various types of cells and extracellular matrix. The fibers of the extracellular matrix consist of proteins (collagen and elastin). The adventitia also consists of nerves and vasa vasorum, the latter being small vessels that supply larger vessels with nutrients and oxygen $[2,3]$.

Elastin and collagen are two different proteins, and have different properties. Elastin fibers are soft and elastic and give blood vessels and other tissues most of their resilient properties [1]. Collagen fibers provide strength and are the major structural component of connective tissues, carrying tension [1]. Collagen is also elastic, but has a much larger Young's modulus than elastin, which makes the collagen stiffer [1]. The vessels' proportion of elastin and collagen varies in the vascular system, for instance the aorta close to the heart is more compliant than more distant parts of the vascular system $[1,2]$. Most blood vessels contain vascular smooth muscle [1]. When the smooth muscle cells contract (vasoconstriction), the diameter of the vessel decreases, whereas when the smooth muscle cells relax (vasodilatation), the diameter increases. This allows vessels to maintain appropriate levels of blood pressure and blood flow. In large arteries, e.g. the aorta, contraction is a more passive process, not due to the work of smooth muscle cells but to the elastic recoil of the vessel after dilatation. 

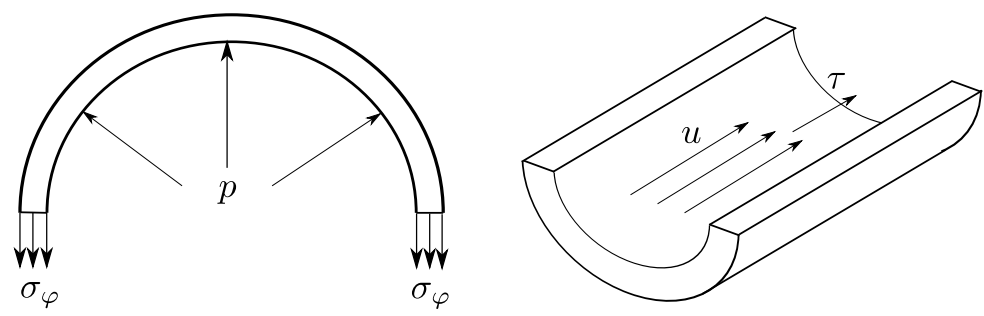

Figure 2: Stresses, blood pressure $p$ and volumetric flow rate $u$ in blood vessels. (a) Circumferential stress $\sigma_{\varphi}$ and (b) shear stress $\tau$.

\subsection{Growth and remodeling in blood vessels}

The heart is a biological pump that ejects blood into the vasculature only during its contractile phase and creates a forward flow in the blood vessel. When the blood comes in pulsating waves the vessels undergo dilation and relaxation. Thus the heart generates the blood pressure $p$ and the volumetric flow rate $u$ in the blood vessels. These mechanical parameters are related to the circumferential stress and shear stress that are created in the vessel wall (see Fig. 2). They affect the growth and remodeling of blood vessels so that they can withstand the pressure and flow, and at the same time maintain a homeostatic state $[4,5,6,7]$. According to Humphrey [8], the term growth refers to an increase in mass, achieved by an increase in the number or size of cells, or by an increase in extracellular matrix. The term remodeling means a change in internal structure. As a result of growth and remodeling the material properties change [8].

The circumferential stress is related to the transmural pressure, the radius of the blood vessel and thickness of the vascular wall. Cells in the vessel wall sense the stress and signal to the growth control system. The shear stress is determined by blood flow, vessel geometry and fluid viscosity. Blood that flows through the vessel shears the endothelium of the vessel wall. Mechanoreceptors on the surface of the endothelial cells sense the shear stress, and through a cascade of molecular signals, the surrounding cells react and the vessel adapts its diameter to the new level of blood flow $[9,10,11]$.

Many experimental studies have been carried out on how the blood pressure and volumetric flow rate affect the growth and remodeling of the blood vessels $[4,5,6,7]$. It has been shown that the thickness of the vessel wall increases as the pressure increases, enabling the vessels to adapt and to maintain a homeostatic state $[5,7]$. 
Some studies also show how the material composition of the vessel wall changes at increased pressure [5]. When flow is increased, the diameter of the blood vessel is increased, so that the shear stress is kept at a homeostatic state [4]. On a longer time scale, the response to increased flow is a change in the composition of the vessel wall [6]. 


\section{Motivation}

In the vascular system of a healthy individual, the living composition of the arterial wall must regenerate and remodel continuously during the entire lifetime to maintain the system and enable it to withstand the stresses it is affected by. In some cases the system destabilizes due to disease, injury or other complex processes [8].

Several different disease states may develop in the vascular system, for instance aneurysms. Abdominal aortic, thoracic and intracranial aneurysms are related to abnormal changes in the composition or morphology of vessels $[12,13]$. The tensile strength of the vessel wall is decreased because of thinning or composition change and this can eventually lead to life-threatening rupture [13]. Aging also affects the vessels, and especially the media: the elastin concentration decreases while the collagen concentration increases and the vessel becomes stiffer $[3,14,15,16]$. In addition to this, elastin fibers and laminae become more disorganized [3]. These processes constitute an abnormal growth and can lead to disease. If the blood vessel is affected by injury, this can lead to inflammation or infection, and that can lead to disease and abnormal growth.

According to the European Cardiovascular Disease Statistics, cardiovascular diseases cause about 47 percent of all deaths in Europe [17]. The number of deaths could be reduced by better clinical treatment and methods to predict the disease at an early stage, for instance in case of abdominal aortic aneurysm (AAA). In the light of this, it is important to develop more efficient engineering tools to predict and to diagnose disease. To make this possible, it is necessary to have a good understanding of how blood vessels function, determining the evolution of the organ morphology and obtain predictive capability under normal conditions as well as in disease. This could be achieved by mathematical modeling of such vessels. Mathematical modeling of the growth control system offers a possibility for stability analyses, and for identifying the cause of instability and associated diseases. Mathematical modeling can also give an understanding of the optimal structure of blood vessels.

Humphrey and co-workers have formulated an evolution equation for mass fractions by letting the mass production in the vessel wall depend on differences between actual and target homeostatic stresses [18, 19, 20, 21, 22]. The mechanical model used in their works is based on constrained mixture theory (CMT), which takes care of production and removal of arterial constituents. This thesis aims to contribute 
to the stability analyses of the growth process by formulating a new goal function approach. This makes it possible to study stability of growth processes in blood vessels and examining under which conditions the instability arises.

Regarding the optimal design of blood vessels, it was Murray who first developed a cost function for the vascular system, by adding the cost of energy required to drive the flow of blood and the metabolic energy required to maintain the blood supply [23]. This cost optimum lead to the formulation of Murray's law, which predicts the radius of the blood vessel:

$$
r \propto u^{1 / 3}
$$

Taber modified Murray's law by adding the metabolic cost of the vessel wall, and by taking into account the passive and active behavior of the vessel [24]. Klarbring [25] and Liu [26] have developed the cost function so that it takes into account the entire vascular tree. These previous studies have not considered how the material composition of the vessel affects the optimum, nor that the material behavior of the blood vessel wall is nonlinear. By using nonlinear material properties, one can describe the material of the blood vessel more realistically. In this study, we aim to analyze changes in the optimum material composition of the vessel wall, using nonlinear material models. 


\section{Methods}

\subsection{Growth and remodeling control system}

Here, we describe how we view the vascular control system. We do not look at the vascular system as a whole, but describe the state of a vessel locally. The local state description includes the composition and geometry of the vessel, collectively denoted by $\Omega$, and the local pressure $p$ and volumetric flow-rate $u$. We assume that there are three different states: the physical state $(\Omega, p, u)$, the apparent state $\left(\Omega^{*}, p^{*}, u^{*}\right)$, and the target state $\left(\hat{\Omega}\left(p^{*}, u^{*}\right), p^{*}, u^{*}\right)$ (see Fig. 3$)$. Note that the target $\hat{\Omega}$ is determined by the apparent flow conditions $\left(p^{*}, u^{*}\right)$.

The control system is not regulated by the physical state in itself, but the control system's perception of the physical state, i.e. the apparent state. The apparent state is formed using information from sensing and signaling processes, for example mechanotransduction of epithelial cells.

After having formed a perception of the state of the vessel (apparent state), the growth and remodeling control system strives to attain a homeostatic state (target state). If the target state is achieved, the vessel will be kept in this state, otherwise the growth process would become unstable. Growth of the vessel is then determined by the difference between the apparent and target states in terms of composition and geometry.

In Paper I, we study the stability of the growth process as the vessel evolves toward a given target state. We assume that the physical state and the apparent state is exactly the same, which means that sensing and signaling processes work perfectly and instantaneously. We then examine under which conditions instability occurs.

In the second paper, we study the cost-optimal target composition and geometry of a vessel given certain levels of blood flow and blood pressure.

\subsection{Thin-walled tube}

We consider the blood vessels as thin-walled tubes. Vessel wall mechanics are an important part of growth and remodeling: the transmural pressure, and the 


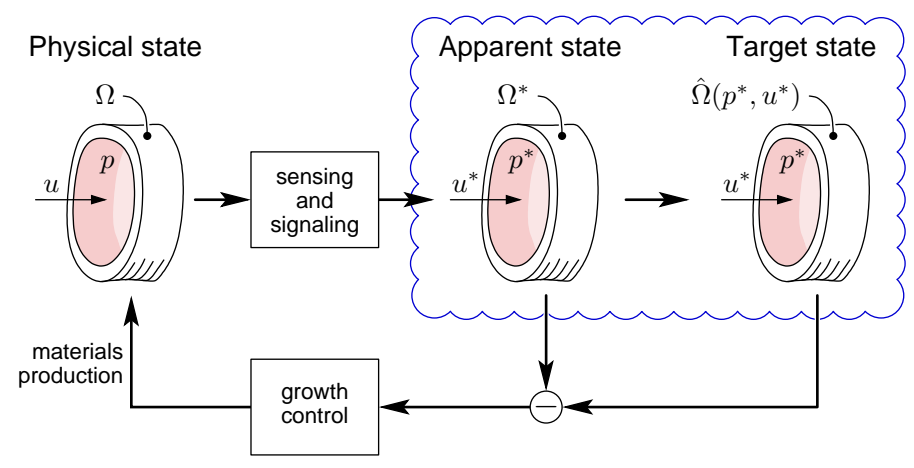

Figure 3: Control system for growth and remodeling. Sensing and signaling processes measure the physical state, $(\Omega, p, u)$, which is perceived as an apparent state, $\left(\Omega^{*}, p^{*}, u^{*}\right)$. The target state is $\left(\hat{\Omega}\left(p^{*}, u^{*}\right), p^{*}, u^{*}\right)$.

vessel geometry and composition are related by an equilibrium equation. This equilibrium equation is derived for thick-walled tubes and assumptions are then made that restrict its applicability to thin-walled tubes (see Paper I):

$$
p=\frac{1}{2 \pi \lambda \lambda_{z} R_{0}^{2}} \frac{\partial}{\partial \lambda} \sum_{k} A^{k} \psi^{k},
$$

where $p$ is the transmural pressure, $R_{0}$ is the reference radius, $A^{k}$ is the effective area of constituent $k, \lambda$ is the stretch in the circumferential direction, $\lambda_{z}$ is the stretch in the axial direction, and $\psi^{k}$ is the strain energy function of constituent $k$.

The equilibrium equation is approximately valid for the time-average pressure and stretch. For more details, see Paper I.

\subsection{Constrained mixture theory}

As a measure of growth and remodeling of a vessel, we study the evolution of effective areas of the vessel wall constituents. In the derivation of the thin-walled tube theory we used CMT to derive the equilibrium equation for a thin-walled tube. CMT assumes that the vessel wall constituents deform together, and therefore undergo the same deformation [21, 22, 27, 28]. The materials of tissues in the body are produced and degraded continually. These mechanisms are accounted for by extending the CMT to include the production and degradation of materials $[19,22]$. The evolution of the constituent effective areas includes two terms: the remaining original materials and the remaining materials $\mathcal{A}^{k} d \tau$ formed at time $\tau$. 
This is formulated as [19]

$$
A^{k}=A^{k}(0) Q^{k}(t)+\int_{0}^{t} \mathcal{A}^{k}(\tau) q^{k}(t-\tau) d \tau, \quad t \geq 0
$$

where $A^{k}(0)$ is the original effective area of constituent $k, Q^{k}(t)$ is the fraction of constituent $k$ that was produced before time 0 and remains at time $t, \mathcal{A}^{k}(t) \geq 0$ is the rate of production of effective area at time $t$ and $q^{k}(t)$ is a monotonically decreasing survival function such that $q(0)=1$.

By assuming that materials created at different time instances contribute to the strain energy in proportion to remaining area fractions, we obtain [19]

$$
\begin{aligned}
A^{k} \psi^{k}(\lambda)= & A^{k}(0) Q^{k}(t) \Psi^{k}\left[\lambda^{k}(t, 0)\right]+ \\
& \int_{0}^{t} \mathcal{A}^{k}(\tau) q^{k}(t-\tau) \Psi^{k}\left[\lambda^{k}(t, \tau)\right] d \tau,
\end{aligned}
$$

where, $\Psi^{k}\left[\lambda^{k}(t, \tau)\right]$ is the strain energy density with respect to a natural, stressfree configuration and characterizes the nonlinear, elastic behavior [19]. There are several kinds of strain energy functions. In this thesis, we use strain energy functions based on references [29] and [30] (see Paper I).

Also, $\lambda^{k}(t, \tau)$ is the stretch at time $t$ for materials produced at time $\tau$. Hence, [19]

$$
\lambda^{k}(t, \tau)=\frac{\lambda(t)}{\lambda(\tau)} G_{\mathrm{h}}^{k}
$$

The ratio $\lambda(t) / \lambda(\tau)$ is the stretch developed between times $\tau$ and $t$, and $G_{\mathrm{h}}^{k}$ is the homeostatic prestretch of constituent $k$. This means that the material can attain prestretch at its time of production.

\subsection{Local remodeling of arteries}

In Paper I, we formulate the goal function in such a way that the constituents grow toward a target potential energy and a target composition

$$
f(\mathbf{A})=\frac{1}{2}[\Pi(\lambda(\mathbf{A}), \mathbf{A})-\hat{\Pi}]^{2}+\frac{1}{2} \sum_{k} b^{k}\left[A^{k}-\hat{A}^{k}\right]^{2},
$$

where $\Pi(\lambda, \mathbf{A})$ is the total potential energy of the system, $\mathbf{A}$ is the vector of effective areas, $\hat{\Pi}$ is the target potential energy, $b^{k}>0$ are constants and $\hat{A}^{k}$ is the target composition.

The total potential energy of the system reads

$$
\Pi(\lambda, \mathbf{A})=\sum_{k} A^{k} \psi^{k}-\pi R_{0}^{2} \lambda_{z} \lambda^{2} p .
$$


The effective area of each constituent is assumed to change in the direction of steepest descent of a goal function. This is given by

$$
\frac{d A^{k}}{d t}=-C \frac{\partial f}{\partial A^{k}}
$$

where $C$ is a positive constant. Also, we do not let the production of effective area become negative.

The evolution equation is nondimensionalized and we find parameters that govern the system's behavior. The parameter $C E_{0}^{2} / \nu^{1}$ controls the first term in the goal function and parameter $b^{1} C / \nu^{1}$ controls the second term. Here, $E_{0}$ denotes the incremental modulus of the vessel wall at the point of operation and $\nu^{1}$ is the degradation rate of collagen and smooth muscle.

As indicated above, it is assumed that the gradient of the goal function governs the evolution toward a homeostatic state. Stability analysis is performed for different types of material models. Here, we look only at the two-constituent model. That is, we divide the blood vessels wall into two components: an isotropic part which does not grow or remodel (elastin, which has a much longer degradation time than other relevant materials, [16]), and an anisotropic part that grows and remodels (collagen and smooth muscle have shorter degradation time [31, 32]). Stability analysis is performed by changing the parameters $C E_{0}^{2} / \nu^{1}$ and $b^{1} C / \nu^{1}$ and then letting the dynamical system evolve toward a target homeostatic state.

\subsection{Target geometry and composition}

In Paper II, we study how the optimal target geometry and composition of a vessel depend on volumetric flow rate $u$, pressure $p$, elastin content $\hat{A}^{\mathrm{e}}$, parameters of the metabolism and parameters related to the material model, when the objective is to optimize the metabolic cost of the vessel at a homeostatic state and at the same time satisfying the equilibrium equation.

We formulate a metabolic goal function (cost function) by extending the theoretical frame of Murray [23], and summing the metabolic cost for the vessel wall, the cost of energy required to drive the flow of blood and the metabolic energy required to maintain the blood supply:

$$
P=\sum_{k} \alpha^{k} \hat{A}^{k}+\beta(R \hat{\lambda})^{2}+\gamma u^{2}(R \hat{\lambda})^{-4}
$$

where $\alpha^{k}, \beta$ and $\gamma$ are constants, $\hat{A}^{k}$ is a time constant composition of materials and $\hat{\lambda}$ is time constant stretch. That is, $\hat{A}^{k}$ and $\hat{\lambda}$ are the target values used in Paper I.

In Paper I, we studied three different material models. In Paper II, we consider only the two-constituent model. 


\section{Results and discussion}

In Paper I, we perform stability analyses for three different material models. Here, we focus on the two-constituent model, which represents the most realistic scenario. In the stability analysis, we have observed three different types of response of the dynamical system: A) when the system converges to a homeostatic state. We call this convergence (Conv.). B) When the system does not reach the homeostatic state, we have two different kinds of response: oscillatory divergence (OD) and monotonic divergence (MD) (see Fig. 4).

By changing the parameters $C E_{0}^{2} / \nu^{1}$ and $b^{1} C / \nu^{1}$ and performing stability analysis, we have mapped the areas in which we get convergence (Conv.), oscillatory divergence (OD) and monotonic divergence (MD) (see Fig. 5). For a detailed discussion of these response areas in relation to the goal function, see Paper I.

The parameters $b^{k}$ and $C$ of the evolution equation influence growth signaling and growth capacity. A decrease in $C$ means a slower response of growth control.

In some disease states, e.g. AAA, inflammation occurs frequently [13,33]. In these cases, degradation of collagen occurs faster than normal, and this degradation is dependent on enzymes called collagenases, e.g. matrix metalloproteinases (MMPs) [13]. This is in agreement with our simulation: as shown in Fig. 5, an increased rate $\nu^{1}$ of collagen degradation leads to an unstable system.

The materials forming the blood vessels become stiffer with increased age $[14,15]$. It is not fully understood if increased stiffness of vessels cause disease, but there is a clear association between atherosclerosis and vessel stiffness [34]. The incremental modulus $E_{0}$ is a measure of stiffness and a stiffening of the vessel wall gives a more stable growth process (see Fig. 5).

When people become older, the elastin concentration of some blood vessels, for instance the ascending thoracic aorta, is reduced [16]. In disease conditions like AAA, the degradation of elastin occurs faster than normal, leading to reduced elastin concentration $[13,16]$. Here, we also study how the elastin concentration affects the growth process. When we remove elastin altogether, the simulation shows that this increases the risk of the growth process becoming unstable (see Paper I and Fig. 6a). We have also studied how the material parameter $c_{1}^{1}$ affects the growth process by increasing it by a factor 2 . This parameter is directly proportional to the stiffness of the material. When $c_{1}^{1}$ is increased, the stability 


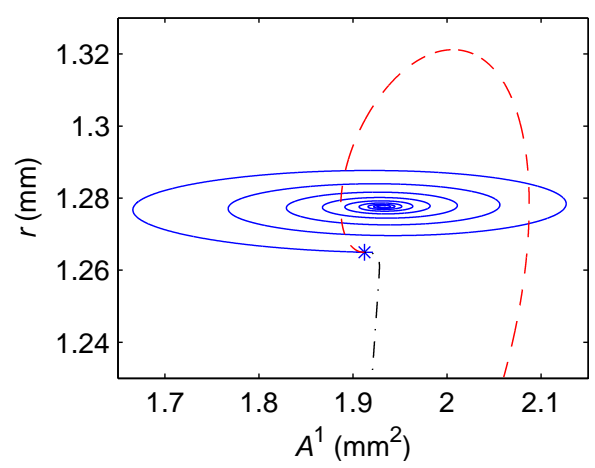

Figure 4: The response of a dynamical system, in terms of geometry and composition, for a two-constituent model. The star represents the starting point of the system. The solid line represents when the system develops and we get convergence (Conv.). The dashed line represents when the system develops and we get oscillatory divergence (OD). The dash-dotted line represents when the system develops and we get monotonic divergence (MD).

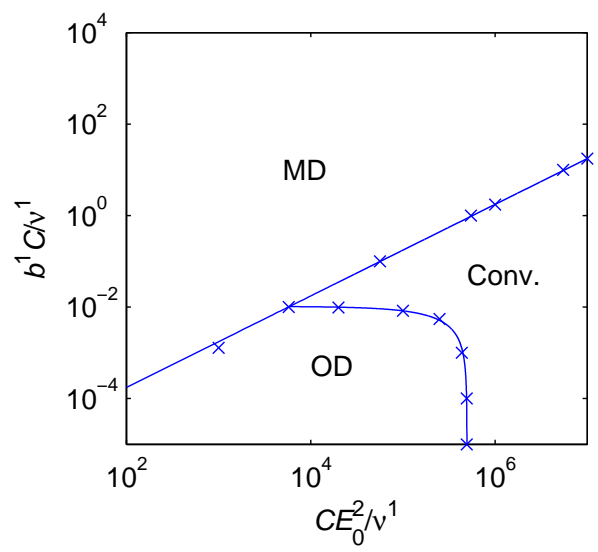

Figure 5: Zones of divergence and convergence for the two-constituent model in a $\left(C E_{0}^{2} / \nu^{1}\right)-\left(b^{1} C / \nu^{1}\right)$ plane. The zones represent monotonic divergence (MD), oscillatory divergence (OD) and convergence (Conv.). 

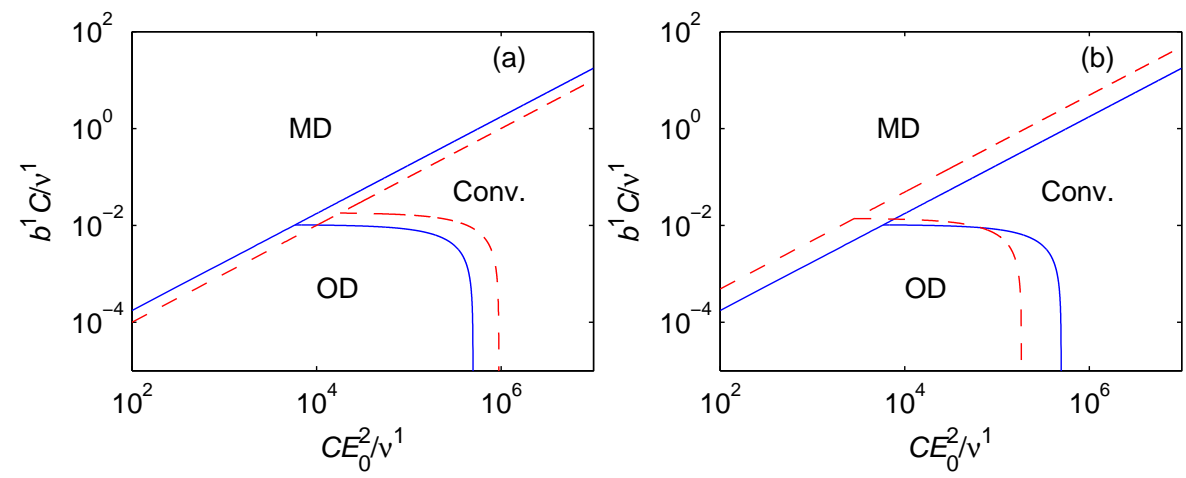

Figure 6: Effects of material properties on growth stability. The solid lines in (a) and (b) represent the two-constituent model for a normal vessel. The dashed line in (a) represents a vessel without elastin. The dashed line in (b) represents a vessel with the material parameter $c_{1}^{1}$ increased by a factor 2 .

analysis shows that the system becomes more stable compared to a healthy vessel (see Fig. 6b and Paper I).

We know from experimental studies that the volumetric flow rate $u$ has an effect on the vessel radius [4] and composition [6]. In Paper II, we investigate how the volumetric flow rate affects the radius and composition when minimizing the metabolic cost of the vessel at a homeostatic state and at same time satisfying the equilibrium equation (see Fig. 7).

The solid line in Fig. 7 represents stationary points of the goal function. If the cost of the vessel wall is assumed to be zero, $\alpha^{\mathrm{t}}=0 \mathrm{~kg} \cdot \mathrm{m}^{-1} \mathrm{~s}^{-3}$, the variations of $r$ with $u$ follow Murray's law. When the metabolic cost of the vessel wall is increased to $\alpha^{\mathrm{t}}=1 \mathrm{~kg} \cdot \mathrm{m}^{-1} \mathrm{~s}^{-3}$ and $\alpha^{\mathrm{t}}=5 \mathrm{~kg} \cdot \mathrm{m}^{-1} \mathrm{~s}^{-3}$ respectively, we get a new series of stationary points (the dashed line and dash-dotted line respectively in Fig. 7). We can also see from Fig. $7 \mathrm{~b}$ that $\hat{A}^{\mathrm{t}}>0$ for all examined values of $u$. For a deeper discussion of the parameters studied, including pressure and elastin content, see Paper II. 

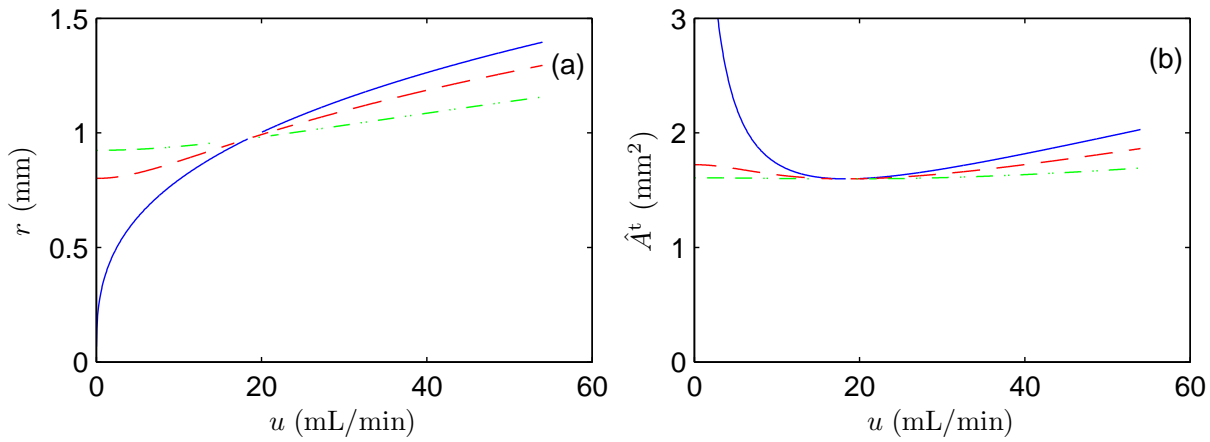

Figure 7: Stationary points of the goal function in (a) the $u-r$ plane and (b) the $u-\hat{A}^{\mathrm{t}}$ plane for different metabolic costs of the vessel wall: $\alpha^{\mathrm{t}}=0 \mathrm{~kg} \cdot \mathrm{m}^{-1} \mathrm{~s}^{-3}$ (solid line), $\alpha^{\mathrm{t}}=1 \mathrm{~kg} \cdot \mathrm{m}^{-1} \mathrm{~s}^{-3}$ (dashed line) and $\alpha^{\mathrm{t}}=5 \mathrm{~kg} \cdot \mathrm{m}^{-1} \mathrm{~s}^{-3}$ (dash-dotted line). 


\section{Conclusions}

The results of our theoretical investigation and simulations show an agreement with factors that are known to lead to disease conditions, for instance AAA. Faster than normal degradation of materials and lower elastin concentration in the vessel wall impair the stability of the growth process. On the other hand, perhaps counter-intuitively, our results also show that increased stiffness of vessels leads to a more stable system. There is a clear association between atherosclerosis and vessel stiffness. However, our results suggests that this increased stiffness may not be a cause of disease, but rather a correlate to it.

On the basis of the principle of minimum work we have extended Murray's law to include growth and remodeling, as well as the nonlinear mechanics of the arterial wall. This gives new insight into the wall's architecture under optimal conditions. 



\section{Bibliography}

[1] W. F. Boron and E. L. Boulpaep. Medical physiology. Saunders, 2008.

[2] J. A. G. Rhodin. Architecture of the Vessel Wall. John Wiley, Sons, Inc., 2011.

[3] W. Nichols, M. O'Rourke, and C. Vlachopoulos. McDonald's Blood Flow in Arteries, Theoretical, Experimental and Clinical Principles. CRC Press, 2011.

[4] R. D. Brownlee and B. L. Langille. Arterial adaptations to altered blood flow. Can. J. Physiol. Pharm, 69(7):978-983, 1991.

[5] T. Matsumoto and K. Hayashi. Stress and strain distribution in hypertensive and normotensive rat aorta considering residual strain. J. Biomech. Eng., 118(1):62-71, 1996.

[6] N. Kubis, A. Checoury, A. Tedgui, and B. I. Lévy. Adaptive common carotid arteries remodeling after unilateral internal carotid artery occlusion in adult patients. Cardiovasc. Res, 50(3):597-602, 2001.

[7] J.-J. Hu, T. W. Fossum, M. W. Miller, H. Xu, J.-C. Liu, and J. D. Humphrey. Biomechanics of the porcine basilar artery in hypertension. Ann. Biomed. Eng., 35(1):19-29, 2007.

[8] J. D. Humphrey. Cardiovascular solid mechanics: cells, tissues, and organs. Springer Verlag, 2002.

[9] S. Lehoux, Y. Castier, and A. Tedgui. Molecular mechanisms of the vascular responses to haemodynamic forces. J. Intern. Med., 259(4):381-392, 2006.

[10] J. Ando and K. Yamamoto. Vascular mechanobiology: Endothelial cell responses to fluid shear stress. Circ. J., 73(11):1983-1992, 2009.

[11] B. D. Johnson, K. J. Mather, and J. P. Wallace. Mechanotransduction of shear in the endothelium: Basic studies and clinical implications. Vasc. Med., 16(5):365-377, 2011.

[12] J. D. Humphrey and G. A. Holzapfel. Mechanics, mechanobiology, and modeling of human abdominal aorta and aneurysms. J. Biomech, 45(5):805-814, 2012 . 
[13] E. Choke, G. Cockerill, W. R. W. Wilson, S. Sayed, J. Dawson, I. Loftus, and M. M. Thompson. A review of biological factors implicated in abdominal aortic aneurysm rupture. Eur. J. Vasc. Endovasc, 30(3):227-244, 2005.

[14] D. Nyhan, J. Steppan, V. Barodka, and D. E. Berkowitz. Vascular stiffness and increased pulse pressure in the aging cardiovascular system. Cardiol. Res. Pract., 1(1):263585, 2011.

[15] S.-J. Lee and S.-H. Park. Arterial ageing. Korean Circ. J., 43(2):73-79, 2013.

[16] A. Tsamis, J. T. Krawiec, and D. A. Vorp. Elastin and collagen fibre microstructure of the human aorta in ageing and disease: A review. $J . R$. Soc. Interface, 10(83):20121004, 2013.

[17] M. Nichols, N. Townsend, P. Scarborough, M. Rayne, J. Leal, and R. LuengoFernandez. European cardiovascular disease statistics, 2012 edition. (4):129, 2012 .

[18] S. Baek, K. R. Rajagopal, and J. D. Humphrey. Competition between radial expansion and thickening in the enlargement of an intracranial saccular aneurysm. J. Elasticity, 80:13-31, 2005.

[19] S. Baek, K. R. Rajagopal, and J. D. Humphrey. A theoretical model of enlarging intracranial fusiform aneurysms. J. Biomech. Eng., 128(1):142-149, 2006.

[20] A. Valentín and J. D. Humphrey. Parameter sensitivity study of a constrained mixture model of arterial growth and remodeling. J. Biomech. Eng., 131(10):101006, 2009.

[21] A. Valentín and J. D. Humphrey. Evaluation of fundamental hypotheses underlying constrained mixture models of arterial growth and remodelling. Philos. T. Roy. Soc. A, 367(1902):3585-3606, 2009.

[22] A. Valentín, L. Cardamone, S. Baek, and J. D. Humphrey. Complementary vasoactivity and matrix remodelling in arterial adaptations to altered flow and pressure. J. R. Soc. Interface, 6(32):293-306, 2009.

[23] C. D. Murray. The physiological principle of minimum work. I. The vascular system and the cost of blood volume. Proc. Natl Acad. Sci. USA, 12(3):207$214,1926$.

[24] L. A. Taber. An optimization principle for vascular radius including the effects of smooth muscle tone. Biophys. J., 74:109-114, 1998.

[25] A. Klarbring, J. Petersson, B. Torstenfelt, and M. Karlsson. Topology optimization of flow networks. Comput. Methods Appl. Mech. Engng, 192:39093932, 2003.

[26] Y. Liu and G. S. Kassab. Vascular metabolic dissipation in Murray's law. Am. J. Physiol-heart. C, 292(3):H1336-H1339, 2007. 
[27] J. D. Humphrey and K. R. Rajagopal. A constrained mixture model for growth and remodeling of soft tissues. Math. Mod. Meth. Appl. S, 12(3):407-430, 2002.

[28] R. L. Gleason and J. D. Humphrey. A mixture model of arterial growth and remodeling in hypertension: Altered muscle tone and tissue turnover. J. Vasc. Res., 41(4):352-363, 2004.

[29] G. A. Holzapfel, T. C. Gasser, and R. W. Ogden. A new constitutive framework for arterial wall mechanics and a comparative study of material models. J. Elasticity, 61:1-48, 2000.

[30] G. A. Holzapfel and R. W. Ogden. Constitutive modeling of arteries. Proc. R. Soc. A, 466:1551-1596, 2010.

[31] R. Nissen, G. J. Cardinale, and S. Udenfriend. Increased turnover of arterial collagen in hypertensive rats. Proc. Natl Acad. Sci. USA, 75(1):451-453, 1978.

[32] G. Martufi and T. C. Gasser. Turnover of fibrillar collagen in soft biological tissue with application to the expansion of abdominal aortic aneurysms. $J$. $R$. Soc. Interface, 9(77):3366-3377, 2012.

[33] E. F. Steinmetz, C. Buckley, and R. W. Thompson. Prospects for the medical management of abdominal aortic aneurysms. Vasc. Endovasc. Surg., 37(3):151-163, 2003.

[34] N. M. van Popele, D. E. Grobbee, M. L. Bots, R. Asmar, J. Topouchian, R. S. Reneman, A. P. G. Hoeks, D. A. M. van der Kuip, A. Hofman, and J. C. M. Witteman. Association between arterial stiffness and atherosclerosis: The Rotterdam study. Stroke, 32(2):454-460, 2001. 
